\title{
Optimizing the configuration of a superconducting photonic band gap accelerator cavity to increase the maximum achievable gradients
}

\author{
Evgenya I. Simakov, Sergey S. Kurennoy, James F. O’Hara, \\ Eric R. Olivas, and Dmitry Yu. Shchegolkov \\ Los Alamos National Laboratory, P.O. Box 1663, Los Alamos, New Mexico 87545, USA
}

(Received 31 July 2013; published 18 February 2014)

\begin{abstract}
We present a design of a superconducting rf photonic band gap (SRF PBG) accelerator cell with specially shaped rods in order to reduce peak surface magnetic fields and improve the effectiveness of the PBG structure for suppression of higher order modes (HOMs). The ability of PBG structures to suppress long-range wakefields is especially beneficial for superconducting electron accelerators for high power free-electron lasers (FELs), which are designed to provide high current continuous duty electron beams. Using PBG structures to reduce the prominent beam-breakup phenomena due to HOMs will allow significantly increased beam-breakup thresholds. As a result, there will be possibilities for increasing the operation frequency of SRF accelerators and for the development of novel compact high-current accelerator modules for the FELs.
\end{abstract}

DOI: 10.1103/PhysRevSTAB.17.022001

PACS numbers: 29.20.Ej, 41.60.Cr, 29.27.-a

\section{INTRODUCTION}

Modern high-power free-electron lasers (FELs) continue to place serious demands on driver accelerators, which are required to provide high current, continuous duty, electron beams with minimal degradation to transverse and longitudinal emittances [1]. Superconducting radio-frequency (SRF) cavities are a natural choice for accelerator systems operating in a continuous wave $(\mathrm{CW})$ mode [2]. The drawback of providing refrigeration for operating the machine IS offset by the relative ease with which $\mathrm{CW}$ radio-frequency (rf) can be coupled into and sustained in SRF cavities. Going to higher frequencies in SRF accelerators will save on cooling power, as well as provide a more compact and lower cost accelerating structure. However, extremely low rf losses in SRF cavities become a handicap when we consider higher-order modes (HOMs), which once excited, oscillate with very high Q-factors and interact with a bunched beam causing instabilities, energy spread, degradation of the beam quality, and additional cryogenic losses $[3,4]$. The beam breakup threshold due to HOM wakefields in the linac scales inversely proportional to frequency squared [5]. Therefore, much of the effort in the field of SRF for high current electron accelerators was directed to ensure strong damping of parasitic HOM oscillations [2,5-7].

To minimize the effects of HOMs, special HOM dampers (absorbers or couplers) are typically attached to the beam

\footnotetext{
*smirnova@lanl.gov

Published by the American Physical Society under the terms of the Creative Commons Attribution 3.0 License. Further distribution of this work must maintain attribution to the author $(s)$ and the published article's title, journal citation, and DOI.
}

tube sections of SRF cavities [2]. The absorber is a section of the beam pipe with a layer of microwave absorbing material (lossy ferrites or ceramics) [8,9]. One of the major disadvantages of absorbers is that ferrite materials are brittle and, if cracked, contaminate SRF cavities with particulate matter. HOM couplers usually are made with either a set of waveguides or coaxial lines connected to the beam pipe. A typical configuration of waveguide couplers uses a Y-shaped arrangement of three waveguides on the beam tube of the accelerating section $[10,11]$. Waveguides act as natural bandpass filters with respect to the fundamental mode and are inherently broadband. However, they often perform inferior to absorbers with respect to sufficiently damping HOMs. Coaxial dampers usually offer stronger damping than waveguide couplers and are more compact [12]. Their main disadvantage is their fundamental rf rejection filters, which must be carefully tuned. In addition to the above, placing HOM couplers or absorbers in beam tubes occupies space on the beam line (in case of the absorbers, outside of the cryostat) which otherwise could be used for accelerating the beam. Therefore, it reduces the fill factor and the real estate gradient. Finally, optimization of the dampers for sufficient absorption of HOMs always leads to compromises with the gradient performance of the accelerating structure. For example, enlarging the beam pipe to facilitate propagation of lowestfrequency HOMs to the absorber lowers the shunt impedance of the fundamental mode.

It has been demonstrated that photonic band gap (PBG) [13] cavities have the intrinsic potential for absorption of HOM power and reduction of wakefields. A PBG accelerating cavity employs a PBG structure in the form of a triangular array of metal rods with one rod removed from the center $[14,15]$. This periodic electromagnetic structure 
possesses a rejection band which serves to confine and localize the fundamental mode around the defect (missing rod) [16]. The spacings of the array and the diameters of rods are adjusted so that the frequencies of HOMs fall outside of the rejection band. In this manner, all parasitic wakefields are not confined at the center and may be extracted at the periphery of the structure [17]. The first room-temperature PBG accelerator cavities were built without an external enclosing wall so that the wakefields freely radiated into surrounding space $[17,18]$. Since SRF cavities are immersed in liquid helium and cooled down to superconducting temperatures, SRF PBG resonators must be enclosed by a solid wall [19]. Coupling waveguides may be attached to the outer wall for efficient extraction of HOM power and also for coupling rf to the fundamental accelerating mode. One possible design for an SRF accelerator section with a PBG cell and couplers is shown in Fig. 1. This accelerator section resonates at the frequency of $2.1 \mathrm{GHz}$ [20]. Two HOM couplers in the form of the WR229 waveguides were placed at the periphery of the PBG cell to reduce the Q-factors of the lowest HOMs. A bigger WR-430 waveguide serves as a high-power fundamentalmode coupler. This design incorporates HOM and fundamental mode couplers as a part of the accelerating structure without losing valuable space on the accelerating line and without causing additional azimuthal asymmetries in the accelerating mode. Together with WR-229 waveguides, the PBG structure serves as an efficient natural rejection filter for the accelerating mode. The WR-229 couplers that are placed in the accelerating cavity provide strong HOM damping without decreasing the shunt impedance of the accelerating mode.

An initial proof-of-principle fabrication of the simplest version of SRF PBG resonator was recently reported in [19]. Two cavities with simple PBG structures made of

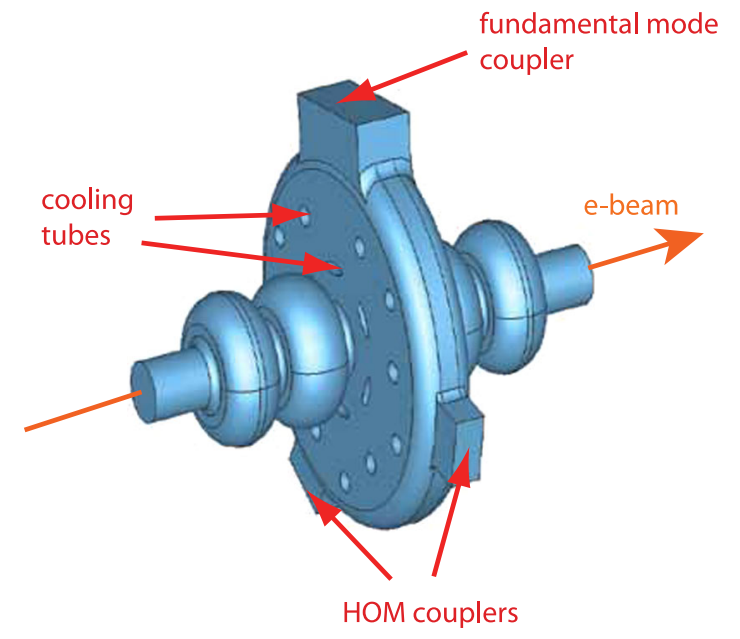

FIG. 1 (color online). A conceptual drawing of an SRF accelerator section consisting of four regular elliptical accelerating cells and an accelerating PBG cell with HOM couplers. round niobium rods were fabricated. The cavities were tested at both 4 and $2 \mathrm{~K}$ and performed well. One demonstrated accelerating gradients of $15 \mathrm{MV} / \mathrm{m}$, which corresponds to peak surface magnetic fields of approximately $130 \mathrm{mT}$. However, in order for SRF PBG cavities to be fully competitive with more traditional SRF coupler configurations for high current accelerator applications, they need to be capable of withstanding the gradients higher than $15 \mathrm{MV} / \mathrm{m}$ without compromising their efficiency with respect to HOM damping.

The research that is reported here presents a novel design of an SRF PBG resonator with elliptically shaped rods, which should be able to maintain gradients 40 percent higher than previously tested resonators with round rods. In addition, this resonator performs better in confining the fundamental accelerating mode and is more efficient for damping HOMs.

\section{MINIMIZATION OF PEAK SURFACE MAGNETIC FIELDS IN A PBG RESONATOR}

We started our investigation of the strategies to minimize peak surface magnetic fields by examining the geometry of the $2.1 \mathrm{GHz}$ SRF PBG resonator with the regular array of round cylindrical rods reported in [19]. The dimensions and peak surface fields in this resonator are summarized in Table I. We investigated ways that the geometry of this structure could be modified so that the peak surface magnetic fields are reduced and overall gradient limitations of the structure are increased. The initial idea was to bend the inner rods of the PBG resonator in a manner mimicking an elliptical SRF cavity, thereby pushing the high magnetic field away from the surface. However, bending the rods of the PBG structure did not produce the same effect (Fig. 2). The surface field reached its maximum value at the point of maximum curvature of the central rod, and this maximum

TABLE I. The dimensions and accelerator characteristics of the $2.1 \mathrm{GHz}$ SRF PBG accelerator cavity with round rods reported in [19].

\begin{tabular}{lc}
\hline \hline Spacing between the rods, $p$ & $56.56 \mathrm{~mm}$ \\
OD of the rods, $d$ & $17.04 \mathrm{~mm}=0.3 \times p$ \\
ID of the rods (cooling channel), $d_{\text {in }}$ & $8.8 \mathrm{~mm}$ \\
ID of the equator, $D 0$ & $300 \mathrm{~mm}$ \\
Thickness of Nb end walls, $t_{\text {wall }}$ & $2.8 \mathrm{~mm}$ \\
Length of the cell, $L$ & $60.73 \mathrm{~mm}=\lambda / 2$ \\
ID of the beam pipe, $R_{b}$ & $31.75 \mathrm{~mm}=1.25 \mathrm{inches}$ \\
Radius of the beam pipe blend, $r_{b}$ & $25.4 \mathrm{~mm}=1 \mathrm{inch}$ \\
Frequency $\left(\mathrm{TM}_{01}\right.$ mode) & $2.100 \mathrm{GHz}$ \\
Geometry factor, $G$ & $179.3 \mathrm{Ohm}$ \\
Ohmic $Q$-factor at $4 \mathrm{~K}, Q_{0}(4 K)$ & $1.5 \times 10^{8}$ \\
Ohmic $Q$-factor at $2 \mathrm{~K}, Q_{0}(2 K)$ & $5.8 \times 10^{9}$ \\
Shunt impedance, $R / Q_{0}$ & $145.77 \mathrm{Ohm}$ \\
$E_{\text {peak }} / E_{\text {acc }}$ & 2.22 \\
$B_{\text {peak }} / E_{\text {acc }}$ & $8.55 \mathrm{mT} /(\mathrm{MV} / \mathrm{m})$ \\
\hline \hline
\end{tabular}



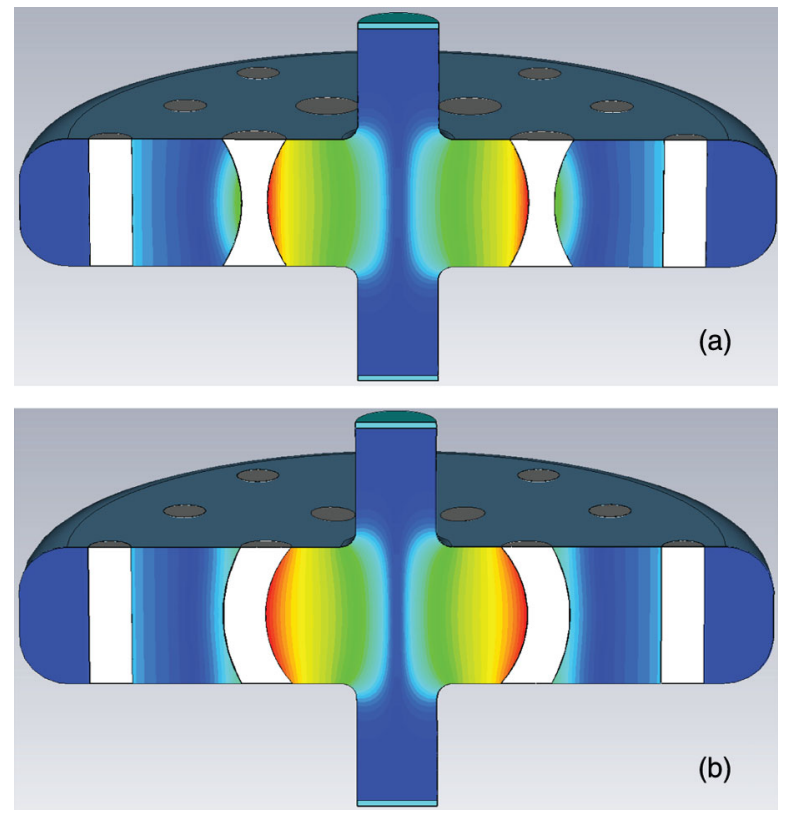

FIG. 2 (color online). Distribution of the magnetic field in a PBG resonator with: (a) central rods with diameter changing along the rod; (b) bent central rods.

value was higher than the peak magnetic field on the surface of an unbent round rod of equal diameter.

Next, we followed the idea of Munroe et al. [21-23] and changed the shape of the six inner rods of the PBG resonator from round cylindrical to an elliptical cross section (Fig. 3). Squeezing the rods in the radial direction towards the center of the resonator produced immediate reduction in peak surface magnetic fields. It is obvious, however, that for very elongated elliptical rods, the magnetic field will increase at the sharp corners of the ellipses. Therefore, the minor radius of the elliptical rods was optimized to minimize the peak surface magnetic field. The optimization was performed for each possible major radius. In the process, we noticed that any changes in the minor radius of elliptical rods resulted in shifting the frequency of the resonator. This had to be compensated for by other changes in the geometry (Fig. 4). One way to adjust the frequency was to put rectangular inserts into each elliptical rod to make the rods thicker [as shown in
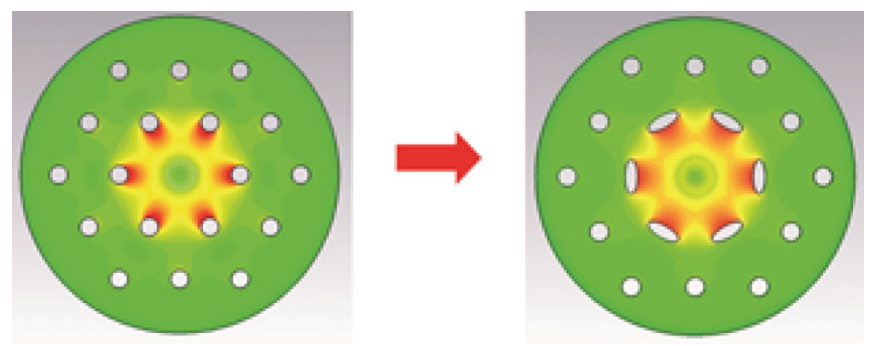

FIG. 3 (color online). Change in the shape of the inner rods of the PBG cavity to reduce peak surface magnetic fields.
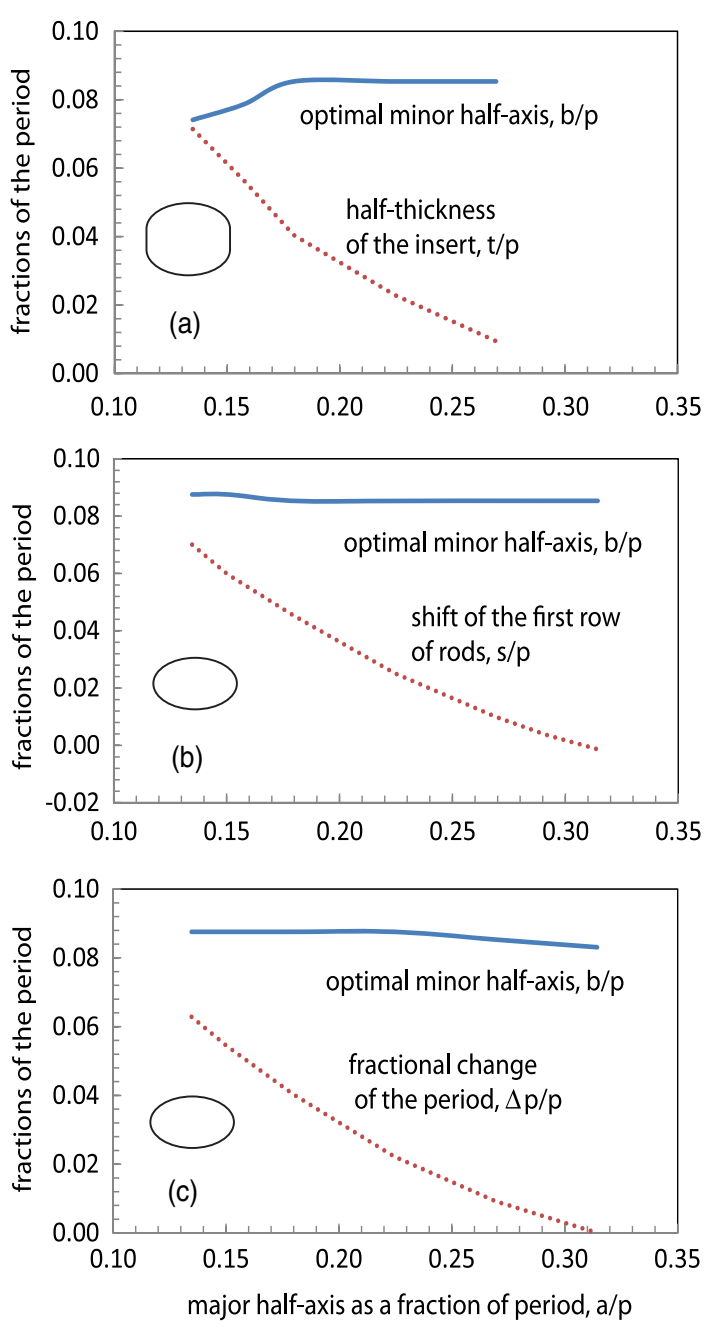

FIG. 4 (color online). Optimized minor half-axis of the elliptical rod and change in dimensions of the PBG structure as a function of the major half-axis of the elliptical rod for: (a) elliptical rods with frequency-compensating rectangular inserts; (b) regular elliptical first row of rods with the rods shifted towards the center of the resonator; (c) regular elliptical first row of rods with the reduced period of the whole PBG structure.

Fig. 4(a)]. Another way was to move the elliptical rods closer to the center of the resonator, and the third way was to decrease the period of the whole PBG structure. The size of the rectangular inserts or the required decrease in the period of the structure are functions of the chosen major radius of the elliptical rod. However, we have discovered that the optimized minor radius stayed nearly constant and almost independent of the major radius and approximately equal to 0.09 of the period of the PBG structure (Fig. 4). Using results from simulations with CST MicrowAVE STUDIO [24], we discovered that if the major half-axis of the new elliptical rod was equal to the radius of the round rod of the resonator of [19] ( 0.15 times the period of the structure) then the peak surface magnetic field immediately decreased by 17 percent. If the major radius of the ellipse is 


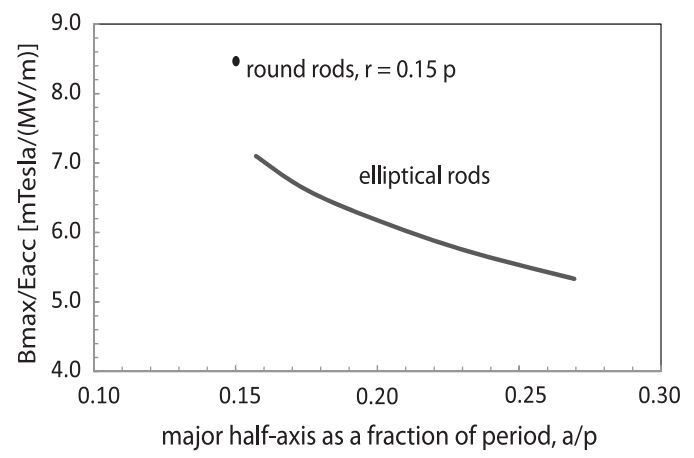

FIG. 5. Dependence of the peak surface magnetic field on the major half-axis of the elliptical rod.

further increased, then the surface magnetic field decreases further below 60 percent of the value in the structure with round cylindrical rods (Fig. 5).

\section{GEOMETRY OPTIMIZATION FOR MAXIMUM HOM SUPPRESSION}

We determined that changing the inner row of PBG rods from the round shape to elliptical shape effectively reduces the peak surface magnetic field. However, a question arises: would the PBG resonator with elliptical rods still be as effective with respect to the confinement of the fundamental mode and suppression of wakefields as the resonator with equally spaced round rods? To characterize the fundamental mode confinement and the filtering of HOMs, we first modeled the PBG resonator with open sidewalls as shown in Fig. 6. We ran simulations with the time-domain solver of the CST MicrowAVE STUDIO and excited the cavity with a virtual current source which was

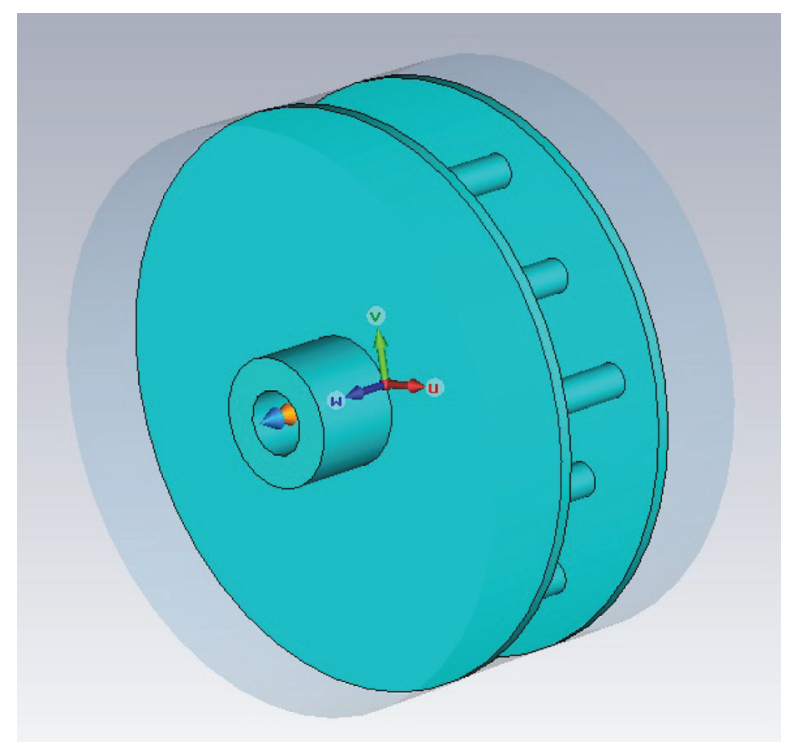

FIG. 6 (color online). CST Microwave STUDIO geometry of a PBG resonator with the open sidewall and excited by a current source.

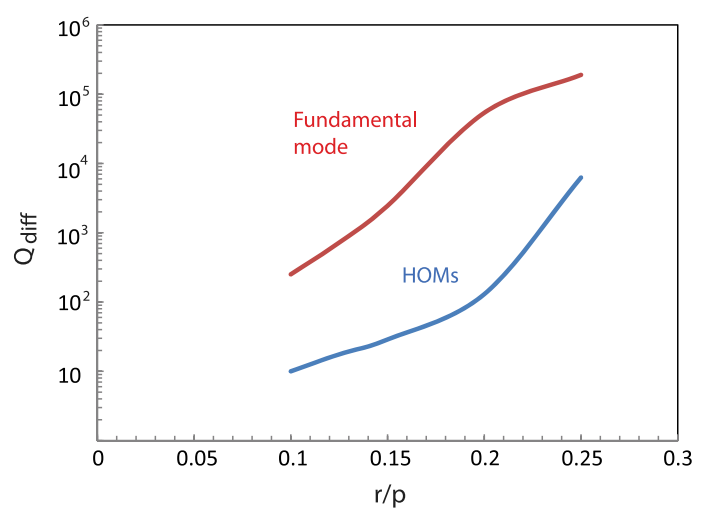

FIG. 7 (color online). A diffraction $Q$-factor for the fundamental mode and the higher order modes in an open PBG resonator made of two rows of equally spaced round rods as simulated with the CST MicrowaVE STUDIO.

placed in a cavity off-axis (at the radius equal to a half of the radius of the beam pipe). The current source was driven by a pulse containing the frequency spectrum of interest. Open boundary conditions were defined in all three directions. We looked at decay rates of microwave energy stored in the cavity and computed the diffraction $Q$-factors of the fundamental mode and the slowest decaying HOM. This method ran fast and converged quickly.

First, we tested the transient method on a well-known geometry and modeled the decay of the fundamental mode and the higher order modes in a cavity with a regular PBG structure of round rods with different diameters. The fundamental mode was excited by a current pulse with a narrow frequency spectrum centered at $2.1 \mathrm{GHz}$. The HOM spectrum was excited with a current pulse with a frequency content from 2.5 to $3.5 \mathrm{GHz}$. The results of this simulation are summarized in Fig. 7. The figure shows the plot of the $Q$-factors for the fundamental mode and the most-slowly decaying HOM as a function of the ratio of the radius of the structure's rod to the period of the structure, $r / p$. It can be seen from the graphs that the decay of both the fundamental mode and the higher order modes slows down as the radii of the rods of the PBG structure increase, and that the fundamental mode is more strongly confined by the structure than the HOMs.

Next, we analyzed the confinement of the fundamental mode and the HOMs in all three types of PBG structure with elliptical rods: rods with rectangular inserts, shifted first row of rods, and the structure with the reduced period. The results are summarized in Fig. 8. The plots show diffraction $Q$-factors computed from the decay of the fundamental mode and HOMs for all three cases. For comparison, the $Q$-factors of the fundamental mode and HOMs for the resonator with round rods and $r / p=0.15$ are shown on each plot. For the particular case of a cavity with the first row of elliptical rods shifted towards the center, it can be seen that for the certain ratios of the elliptical rod's major half-axis to the period of the structure 

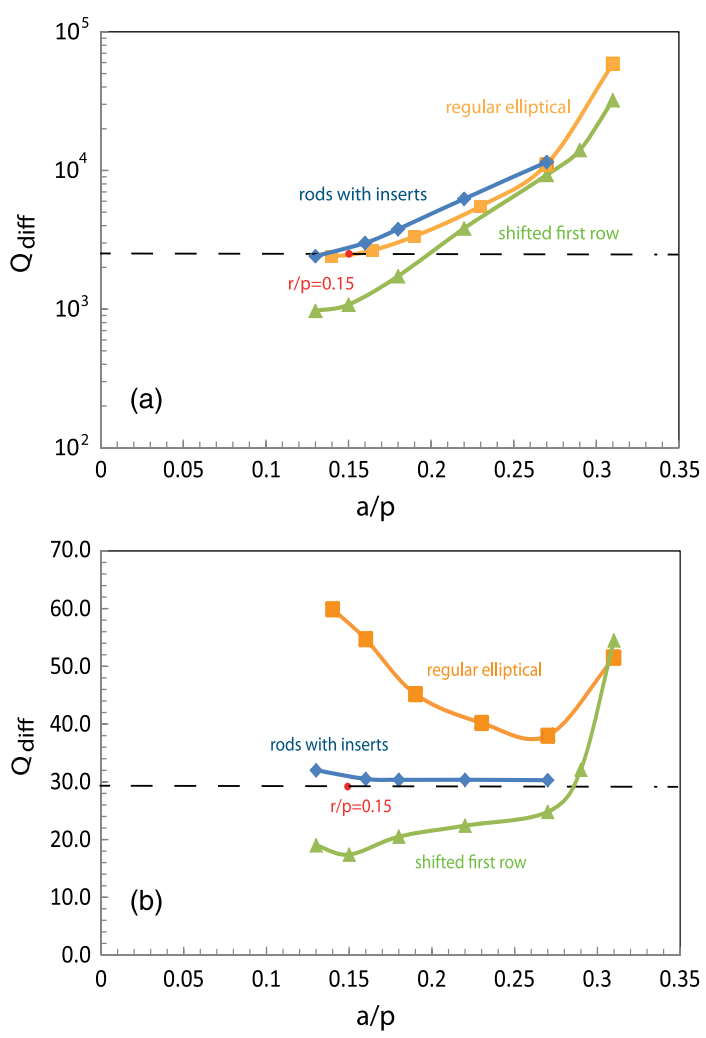

FIG. 8 (color online). A diffraction $Q$-factor for the fundamental mode (a) and the higher order modes (b) in a PBG resonator made of two rows of rods as computed by the CST Microwave STUdio. The six central rods have elliptical shapes and the three cases correspond to elliptical rods with rectangular inserts, elliptical rods shifted to the center, and reduced period of the whole structure. For comparison also shown are the Q-factors of the fundamental mode and HOMs in a structure with equally spaced round rods and $r / p=0.15$.

$a / p<0.27$, the HOMs decay faster than HOMs in the resonator with round rods and $r / p=0.15$. However, for the same structure and $a / p>0.21$, the fundamental mode decays slower than in the structure with round rods and $r / p=0.15$. This effect can probably be explained by the perturbed periodicity of the structure. Together with the fact that the peak surface magnetic fields in the structure with elliptical rods with $0.21<a / p<0.27$ are approximately 40 percent lower than in the structure with round rods, it makes the resonator with elliptical rods shifted towards the center a perfect candidate for a structure with improved wakefield suppression and capability to achieve high accelerating gradients.

To complete the higher-order-mode analysis we modeled a PBG resonator with shifted elliptical rods and $a / p=$ 0.25 surrounded by a solid metal wall with three coupling WR-229 waveguides attached to the wall (Fig. 9). We varied the positions of the three waveguides to achieve the optimal coupling to HOMs. The final angular positions of the waveguides are shown in Fig. 10. The decay of the

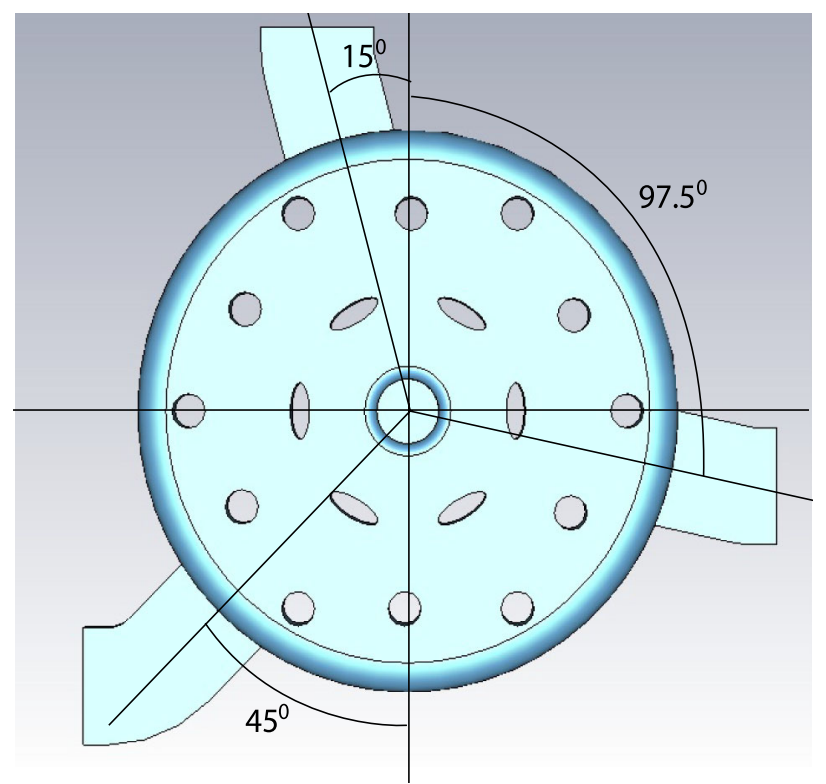

FIG. 9 (color online). The PBG resonator with six elliptical rods and three WR-229 attached for coupling out HOMs.

stored electromagnetic energy in this geometry for frequencies from 2.75 to $3.5 \mathrm{GHz}$ is shown in Fig. 10 . All HOMs decay out through the waveguides with Q-factors below 115.

\section{Thermal analysis}

Thermal analyses were conducted to rule out the possibility of thermal quench due to inadequate cooling. The niobium rods were intended to be manufactured as tubes so that they could be cooled with liquid helium along the length of the rod [19]. High gradient tests were planned to be conducted in a vertical cryostat $965 \mathrm{~mm}$ in diameter and $3048 \mathrm{~mm}$ in depth. The exact geometry was reproduced in the thermal simulations. Thermal analysis was performed

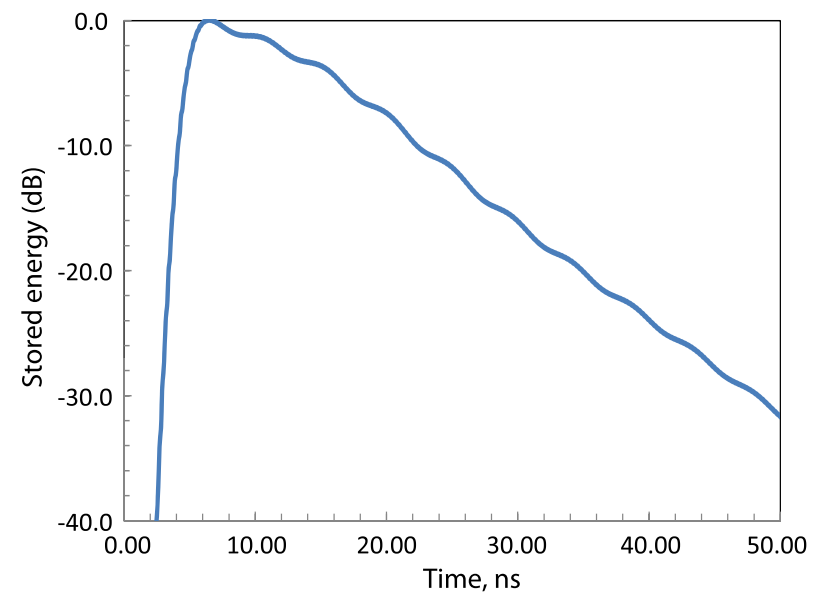

FIG. 10 (color online). The decay of electromagnetic energy through the waveguides of a PBG resonator shown in Fig. 9. 


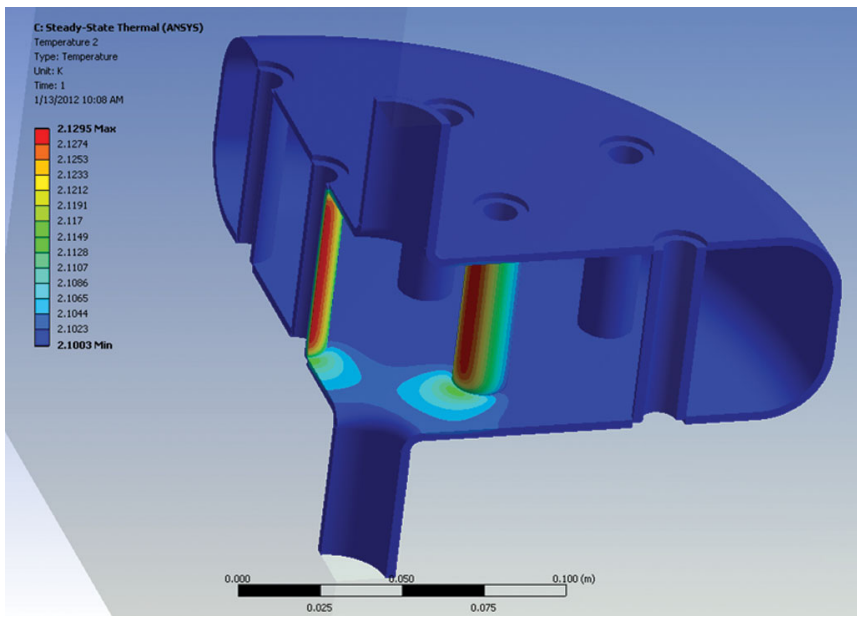

FIG. 11 (color online). Temperature distribution on the surface of a $2.1 \mathrm{GHz}$ SRF PBG resonator with round rods immersed into superfluid liquid helium at 2 kelvin when operating $\mathrm{CW}$ at $10 \mathrm{MV} / \mathrm{m}$ accelerating gradient.

using ANSYS software [25]. The electric and magnetic fields in a PBG resonator operating at a gradient of $10 \mathrm{MV} / \mathrm{m}$ were computed with the CST MiCROWAVE STUDIO and then exported to ANSYS on a compatible grid. Analysis was performed first at 2 kelvin with liquid helium in a superfluid state and then at 4 kelvin when both conduction and free convection mechanisms of heat transfer had to be taken into account.

At 2 kelvin and $10 \mathrm{MV} / \mathrm{m}$ accelerating gradient the estimated thermal load on a whole cavity with round rods was $0.61 \mathrm{~W}$. Due to the high thermal conductivity of superfluid helium at 2 kelvin, the bath did not exhibit any temperature gradient that would drive buoyancy forces (i.e., natural convection), so the heat transfer was purely due to conduction. The temperature distribution on the surface of a niobium cavity with round rods immersed in superfluid helium is shown in Fig. 11. It can be seen from the figure that for $10 \mathrm{MV} / \mathrm{m} \mathrm{CW}$ accelerating gradient the temperature change at the cavity's surface was less than 0.03 kelvin. Therefore, we concluded that the thermal quench is not an issue at 2 kelvin for reasonable accelerating gradients.

At 4 kelvin and $10 \mathrm{MV} / \mathrm{m}$ accelerating gradient the estimated thermal load on a cavity with round rods was $23.6 \mathrm{~W}$ and for the cavity with elliptical rods was $19.28 \mathrm{~W}$. Thermal conductivity of liquid helium at 4 kelvin is approximately seven times lower than at 2 kelvin; therefore, both heat transfer mechanisms were taken into account to accurately simulate cooling of the cavity. The full computational fluid dynamics analysis was conducted with realistic coupling between the two heat transfer mechanisms. The results of this simulation are shown in Fig. 12. It can be seen from that figure that for the cavity with round rods running $\mathrm{CW}$ at $10 \mathrm{MV} / \mathrm{m}$ the peak surface temperature does not exceed 6.72 kelvin, and for the cavity
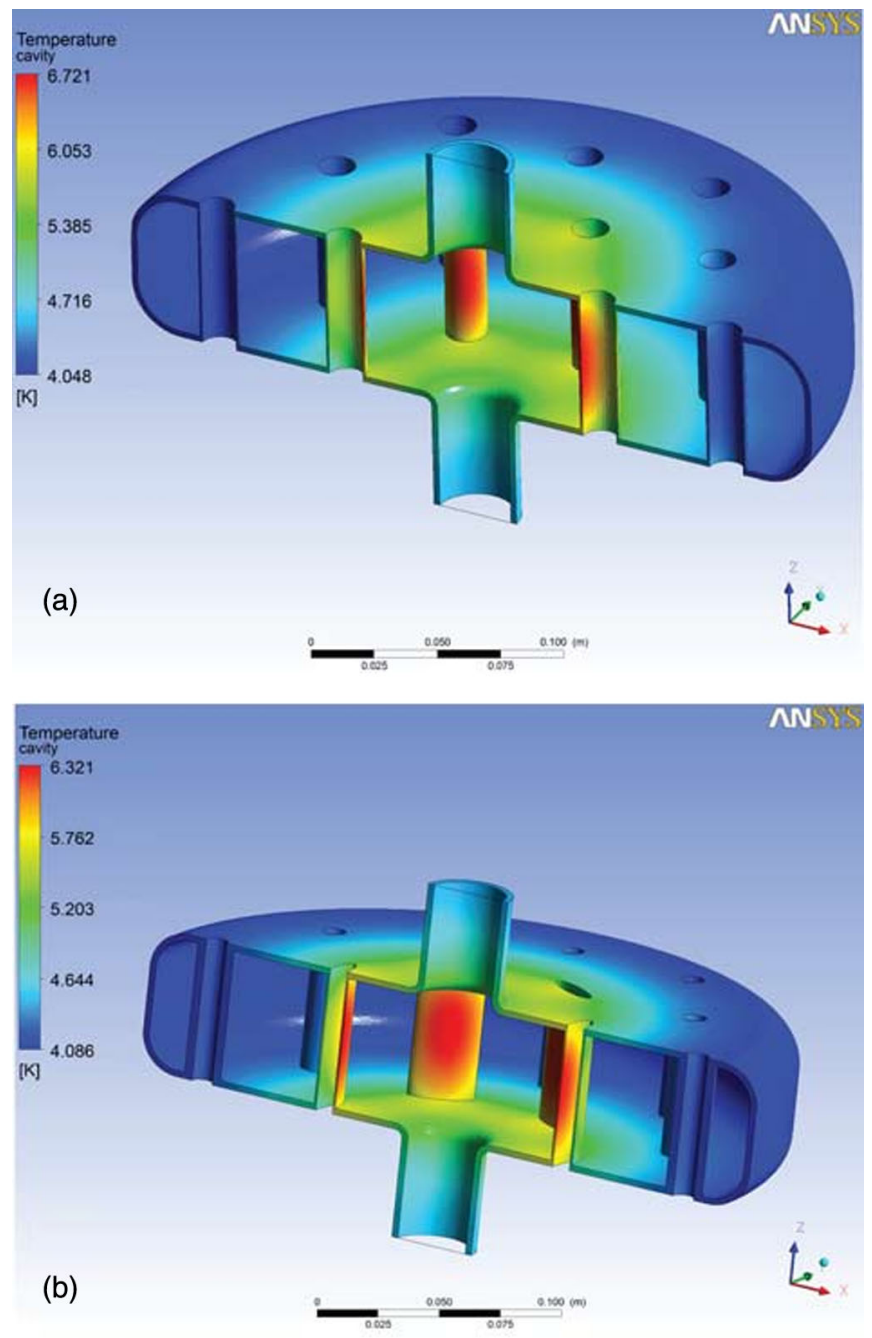

FIG. 12 (color online). Temperature distribution on the surface of a $2.1 \mathrm{GHz}$ SRF PBG resonator immersed into liquid helium at 4 kelvin when operating $\mathrm{CW}$ at $10 \mathrm{MV} / \mathrm{m}$ accelerating gradient: (a) resonator with round rods; (b) resonator with elliptical rods.

with elliptical rods the temperature is somewhat lower, below 6.32 kelvin. Therefore, we concluded that in the tests of the cavity with round rods [19] thermal quench could not be an issue. Maximum observed gradients at 4 kelvin did not exceed $10.6 \mathrm{MV} / \mathrm{m}$, and therefore peak surface temperatures were well below the critical temperature of niobium, which is 9.2 kelvin. However, if the cavity with elliptical rods goes to significantly higher gradients and thermal loads, thermal quench may become an issue, since heating of the surface could become significant.

\section{CONCLUSION}

We have designed an improved 2.1 GHz SRF PBG resonator with six elliptical inner rods. This new resonator is superior to the resonator with round rods due to more efficient higher order mode suppression and better high gradient performance. The improved resonator has 40 
percent lower peak magnetic fields than the resonator with round rods. We simulated the damping of HOMs in this resonator with three WR-229 waveguides attached to the outside metal wall and found superior performance. We have conducted thermal analysis and concluded that thermal quench should not be an issue in this geometry at 2 kelvin, but may become an issue at 4 kelvin if the resonator achieves gradients significantly higher than $10 \mathrm{MV} / \mathrm{m}$ without experiencing magnetic quench. The resonator with elliptical rods has recently been fabricated with niobium. It is currently undergoing high power tests at Los Alamos National Laboratory. Maximum gradients achieved experimentally will be reported elsewhere.

\section{ACKNOWLEDGMENTS}

This work was supported by the Department of Defense High Energy Laser Joint Technology Office through the Office of Naval Research. The authors gratefully acknowledge discussions with Sergey A. Arsenyev, W. Brian Haynes, Frank L. Krawczyk, Tsuyoshi Tajima, Chase H. Boulware, and Terry L. Grimm.

[1] National Research Council of the National Academies, Scientific Assessment of High-power Free-electron Laser Technology (National Academies Press, Washington, DC, 2009).

[2] S. Belomestnykh, Rev. Accel. Sci. Techol. 05, 147 (2012).

[3] J. Sekutowicz, Proceedings of LINAC06, 2006 (Joint Accelerator Conference Website (JACoW), Genewa, Switzerland), p. 506.

[4] R. A. Rimmer, Nucl. Instrum. Methods Phys. Res., Sect. A 557, 259 (2006).

[5] G. H. Hoffstaetter and I. Bazarov, Phys. Rev. ST Accel. Beams 7, 54401 (2004).

[6] G. H. Hoffstaetter and Y.H. Lau, Phys. Rev. ST Accel. Beams 11, 070701 (2008).

[7] G. H. Hoffstaetter and B. Buckley, in Proceedings of the 22nd Particle Accelerator Conference, Albuquerque, New Mexico, econf THPASO43 (2007).

[8] E. Chojnacki, T. Gruber, N. Sherwood, and W. J. Alton, in Proceedings of the 18th Particle Accelerator Conference, New York, 1999 (IEEE, New York, 1999), p. 845.

[9] T. Tajima, K. Asano, T. Furuya, M. Izawa, S. Mitsunobu, T. Takahashi, N. Gamo, S. Iida, Y. Ishi, Y. Kijima, S. Kokura, M. Kudo, K. Sennyu, S. Tachibana, H. Takashina, and N. Taniyama, in Proceedings of the Particle Accelerator
Conference, Dallas, TX, 1995 (IEEE, New York, 1995), p. 1620.

[10] R. A. Rimmer, R. Bundy, G. Cheng, G. Ciovati, E. F. Daly, R. Getz, J. Henry, W. R. Hicks, P. Kneisel, S. Manning, R. Manus, F. Marhauser, K. Smith, M. Stirbet, L. Turlington, L. Vogel, H. Wang, and K. M. Wilson, in Proceedings of the 22nd Particle Accelerator Conference, Albuquerque, New Mexico, 2007, econf WEPMS068 (2007).

[11] F. Marhauser, W. Clemens, G. Cheng, G. Ciovati, E. F. Daly, J. Forehand, J. Henry, P. Kneisel, S. Manning, R. Manus, R. A. Rimmer, C. Tennant, H. Wang, and K. M. Wilson, in Proceedings of the 11th European Particle Accelerator Conference, Genoa, 2008, econf. MOPP140 (2008).

[12] A. Butterworth, P. Brown, O. Brunner, E. Ciapala, H. Frischholz, G. Geschonke, E. Peschardt, and J. Sladen, Nucl. Instrum. Methods Phys. Res., Sect. A 587, 151 (2008).

[13] E. Yablonovitch, Phys. Rev. Lett. 58, 2059 (1987).

[14] D. R. Smith, S. Schultz, N. Kroll, M. Sigalas, K. M. Ho, and C. M. Souloulis, Appl. Phys. Lett. 65, 645 (1994).

[15] D. R. Smith, D. Li, D. C. Vier, N. Kroll, S. Schultz, and H. Wang, AIP Conf. Proc. 398, 518 (1997).

[16] E. I. Smirnova, C. Chen, M. A. Shapiro, J. R. Sirigiri, and R. J. Temkin, J. Appl. Phys. 91, 960 (2002).

[17] E. I. Smirnova, I. Mastovsky, M. A. Shapiro, R. J. Temkin, L. M. Earley, and R. L. Edwards, Phys. Rev. ST Accel. Beams 8, 091302 (2005).

[18] E. I. Smirnova, A. S. Kesar, I. Mastovsky, M. A. Shapiro, and R. J. Temkin, Phys. Rev. Lett. 95, 074801 (2005).

[19] E. I. Simakov, W. B. Haynes, M. A. Madrid, F. P. Romero, T. Tajima, W. M. Tuzel, C. H. Boulware, and T. L. Grimm, Phys. Rev. Lett. 109, 164801 (2012).

[20] S. A. Arsenyev and E. I. Simakov, AIP Conf. Proc. 1507, 425 (2012).

[21] R. A. Marsh, B. J. Munroe, M. A. Shapiro, and R. J. Temkin, in Proceedings of the U.S. Particle Accelerator Conference, 2009 (Joint Accelerator Conference Website (JACoW), Genewa, Switzerland) econf WE6RFP081.

[22] B. J. Munroe, R. A. Marsh, M. A. Shapiro, and R. J. Temkin, in Proceedings of the International Particle Accelerator Conference, Kyoto, Japan, 2010, econf THOD063 (2010).

[23] B. J. Munroe, A. M. Cook, M. A. Shapiro, R. J. Temkin, V. A. Dolgashev, L. L. Laurent, J. R. Lewandowski, A. D. Yeremian, S. G. Tantawi, and R. A. Marsh, Phys. Rev. ST Accel. Beams 16, 012005 (2013).

[24] Computer Simulation Technology, CST Microwave STUDIO, http://www.cst.com.

[25] ANSYS, Ansys Inc., http://www.ansys.com. 